\title{
CFD Analysis of the Effect of Elbow Radius on Pressure Drop in Multiphase Flow
}

\author{
Quamrul H. Mazumder \\ Mechanical Engineering, University of Michigan-Flint, Flint, MI 48502, USA \\ Correspondence should be addressed to Quamrul H. Mazumder, qmazumde@umflint.edu \\ Received 9 April 2012; Accepted 24 September 2012 \\ Academic Editor: Aiguo Song \\ Copyright ( $) 2012$ Quamrul H. Mazumder. This is an open access article distributed under the Creative Commons Attribution \\ License, which permits unrestricted use, distribution, and reproduction in any medium, provided the original work is properly \\ cited. \\ Computational fluid dynamics (CFD) analysis was performed in four different 90 degree elbows with air-water two-phase flows. \\ The inside diameters of the elbows were $6.35 \mathrm{~mm}$ and $12.7 \mathrm{~mm}$ with radius to diameter ratios $(r / D)$ of 1.5 to 3 . The pressure \\ drops at two different upstream and downstream locations were investigated using empirical, experimental, and computational \\ methods. The combination of three different air velocities, ranging from 15.24 to $45.72 \mathrm{~m} / \mathrm{sec}$, and nine different water velocities, \\ in the range of $0.1-10.0 \mathrm{~m} / \mathrm{s}$, was used in this study. CFD analysis was performed using the mixture model and a commercial code, \\ FLUENT. The comparison of CFD predictions with experimental data and empirical model outputs showed good agreement.
}

\section{Introduction and Background}

In most industrial processes, fluids are used as a medium for material transport. A complete knowledge of the principles that rule the phenomena involving fluids transportation leads to more efficient and secure systems. However, in many industries, such as petroleum, chemical, oil, and gas industries, two-phase or multiphase flow is frequently observed [1]. Multiphase flow is defined as the simultaneous flow of several phases, with the simplest case being a twophase flow [2]. Compared to single-phase flow, the equations associated with two-phase flow are very complex, due to the presence of different flow patterns in gas-liquid systems [3]. A detailed discussion of two-phase flow phenomenon behavior is provided by Wallis [2]. The flow patterns observed in horizontal flow are bubble, stratified, stratified wavy, slug, and annular. In vertical flows, bubble, plug, slug (or churn), annular, and wispy-annular flow patterns are present. Several investigations have been reported to determine the friction factor and pressure drops in horizontal [4] and vertical [5] two-phase and multiphase flows [6]. The presence of the two-phase flow typically produces an undesirable higherpressure drop in the piping components. In most industrial installations, elbows are frequently used to direct the flow and provide flexibility to the system [7]. Since these fittings are also used to install instruments that monitor the main parameters of the industrial process, it is important to have a reliable way to evaluate the pressure drop in these elbows [8]. As the fluid flows through the bend, the curvature of bend causes a centrifugal force; the centrifugal force is directed toward the outer wall of the pipe from the momentary center of the curvature. The combined presence of centrifugal force and boundary layer at the wall produces the secondary flow, organized, ideally, in two identical eddies. This secondary flow is superimposed to the mainstream along the tube axis, resulting in a helical shape streamline, flowing through the bend [9].

One of the challenges with undesirable higher-pressure drop is the difficulty in determining a model for twophase flow through pipe components. Despite unsuccessful attempts to develop an accurate model for two-phase flow through pipe components, Chisholm [10] presented an elementary model for prediction of two-phase flow in bends, based on a liquid two-phase multiplier, for different pipe diameters, $r / D$ values, and flow rates. Detailed studies of two-phase pressure loss have largely been confined to 
the horizontal plane. Chenoweth and Martin [11] showed that while the two-phase pressure drop around bends was higher than in single-phase flow, it could be correlated by an adoption of the Lockhart-Martinelli [12] model, a model initially developed for straight pipe. The correlation claimed to predict loss in bends and other pipe fittings. Also, at high mass velocities, agreement was achieved with the homogeneous model. Fitzsimmons [13] presented twophase pressure loss data for bend in terms of the equivalent length and the ratio of the bend pressure loss to the straight pipe frictional pressure gradient; the Lockhart-Martinelli multiplier referred to the single-phase gas pressure loss in the bend. The comparison against pressure drop in straight pipe gave a poor correlation. Sekoda et al. also used a two-phase multiplier, referred to as a single-phase liquid pressure loss in the bend. The two-phase bend pressure drop was found to be dependent on the $r / D$ ratio, while being independent of pipe diameters [14]. The main focus of this paper is to investigate pressure drop for two-phase air-water mixture flow in 90 degree vertical to horizontal elbows. The first step is the prediction of the flow pattern, and then proposing an associated method of calculating the liquid holdup, which is used to determine the twophase friction factor. Comparative studies proved that these models are inconsistently performed, as flow conditions vary. Therefore, the selection of the most appropriate flow correlation is very important in this study [15]. Reported work on the orientation of the plane of the bend has often given contrary results. Debold [16] claimed that the horizontal bend, the horizontal to vertical upbend, and the vertical down to horizontal bend all gave the same bend pressure loss. However, a horizontal to vertical downbend had a pressure drop that was 35\% less. The correlation for elevation was assumed to follow the homogeneous model by Debold [16], but others, such as Alves [17], ignored head pressure differences entirely. Peshkin [18] reported that horizontal to vertical downflow had about $10 \%$ more bend pressure drop than the corresponding horizontal to vertical upflow case. Kutateladze [19], by contrast, concluded the direct opposite: that the horizontal to vertical upflow bend created the greater pressure drop. Moujaes and Aekula reported the effects of pressure drop on turning vanes in 90 degree duct elbows, using CFD models in HVAC applications area [20]. Due to the different approaches that can be used to predict pressure drop in elbows, the current study uses CFD analysis in four different elbows. The CFD analysis results were validated using two different empirical models by Azzi and Friedel [9] and Chisholm, [10] as well as experimental data.

\section{The CFD Approach}

Due to the advancements in computer hardware and software in recent years, the computational fluid dynamics technique has been a powerful and effective tool to understand the complex hydrodynamics of gas-liquid two-phase flows. The commercial CFD package, FLUENT, was used to model the air-water flow, in order to predict pressure drop in 90 degree elbows.
TABLE 1: Configurations of elbows used in the study.

\begin{tabular}{lccc}
\hline & $\begin{array}{c}\text { Pipe diameter, } \\
D(\mathrm{~mm})\end{array}$ & $\begin{array}{c}\text { Elbow curvature } \\
\text { radius, } r(\mathrm{~mm})\end{array}$ & $\begin{array}{c}\text { Equivalent pipe } \\
\text { length, } \mathrm{Le}(\mathrm{mm})\end{array}$ \\
\hline Elbow 1 & 12.7 & 38.1 & 635 \\
Elbow 2 & 12.7 & 19.05 & 571.5 \\
Elbow 3 & 6.35 & 19.05 & 317.5 \\
Elbow 4 & 6.35 & 9.525 & 285.75 \\
\hline
\end{tabular}

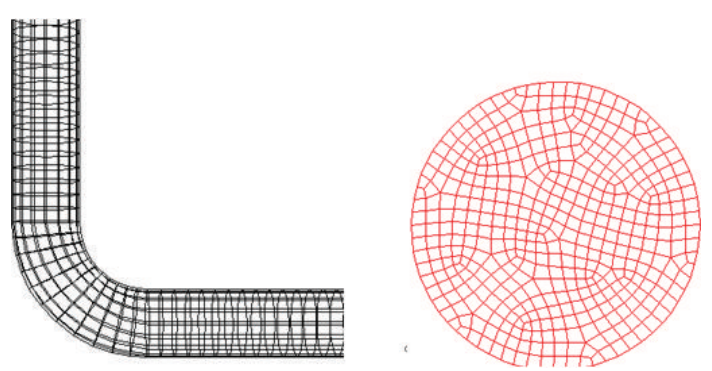

FIgURE 1: Elbow mesh with inlet domain.

\section{Geometry Details}

To conduct this study, four three-dimensional 90 degree vertical to horizontal elbows were created using GAMBIT. The geometries were then imported to FLUENT to simulate pressure drop. Table 1 lists the pipe diameter $(D)$, elbow curvature radius $(r)$, and equivalent pipe length $(L e)$, for different elbows.

The $r / D$ ratio of 1.5 to 3 was used to represent the standard short- and long-radius elbows. A straight pipe section of 45-50 times the pipe diameter was added at both upstream and downstream of the elbow. The Le/D ratio for single-phase flow is typically 10 . However, for the two-phase flow, an Le/D of approximately 100-150 is required for fully developed flow [21]. Due to limitations of the experimental test system, the $L e / D$ ratio used in this study was 50 for elbows 1 and 3, while the ratio was 45 for elbows 2 and 4. Structured mesh was used with the optimum number of nodes. A mesh independency study was performed to determine reasonable results that are independent of the size of the grid. Hexahedral mesh was used, due to its capabilities in providing high-quality solution, with a fewer number of cells than comparable tetrahedral mesh for simple geometry [22]. Figure 1 shows the elbow and inlet domain of threedimensional mesh for elbow 1 .

Table 2 lists the mesh details for each elbow, generated in GAMBIT, to be used in this study. CFD Analysis results at four different locations in the upstream and the downstream of the elbow were used in this study, as shown in Figure 2.

\section{Multiphase Modeling}

The numerical calculations of multiphase flows can be calculated through two approaches: the Euler-Euler approach and the Euler-Lagrange approach. Using the Euler-Euler approach is more efficient, because the different phases are 
TABLE 2: Mesh details of all four elbows.

\begin{tabular}{lccc}
\hline & $\begin{array}{c}\text { Number of } \\
\text { nodes }\end{array}$ & $\begin{array}{c}\text { Number of wall } \\
\text { faces }\end{array}$ & $\begin{array}{c}\text { Number of } \\
\text { hexahedral cells }\end{array}$ \\
\hline Elbow 1 & 110,558 & 33,344 & 92,738 \\
Elbow 2 & 97,520 & 29,408 & 81,791 \\
Elbow 3 & 21,484 & 8,368 & 16,736 \\
Elbow 4 & 18,942 & 7,376 & 14,752 \\
\hline
\end{tabular}

treated mathematically, as interpenetrating continua. The concept of phasic volume fraction is used in this approach, since the volume of phase cannot be occupied by the other phases. These volume fractions are assumed to be continuous functions of space and time, and their sum is equal to one. For each phase, conservation equations are derived to obtain a set of new equations which have similar structure for all phases. By providing constitutive relations obtained from the empirical information, these sets of equations are closed. There are three available Euler-Euler multiphase models in the fluent commercial code: the Eulerian model, the mixture model, and the volume of fluid model. In this study, the mixture model is used, as it is relatively easy to understand for multiphase modeling.

\section{Mixture Model Theory}

The mixture model can be used to model multiphase flows, by assuming the local equilibrium over short spatial length scales, and where the phases move at different velocities. In addition, the mixture model can be used to calculate nonNewtonian viscosity. It can model a number of phases by solving the momentum, continuity, and energy equations for the mixture, the volume fraction equation for the secondary phases, and algebraic expression for the relative velocities. The mixture model is a good substitute for the full Eulerian multiphase model in several cases, as the full multiphase model may not be feasible, due to the wide distribution of a particular phase, when the interphase laws are unknown, or when the reliability of interphase laws is questioned. While solving a smaller number of variables than the full multiphase model, the mixture model can perform as well as the full multiphase model. It uses a single-fluid approach, just like the volume of fluid model but allows for the phases to be interpenetrating and for the phases to move at the different velocities, using the concept of slip velocities.

\section{Mathematical Formulation}

The mixture models solve the continuity equation, momentum equation, energy equation, the volume fraction equation for the secondary phases, and the algebraic expression for the relative velocities, since the two phases are moving at different velocities. The continuity, momentum, and relative velocity equations used in the mixture model are shown in the following section.

The mixture's continuity equation is given by

$$
\frac{\partial}{\partial t}\left(\rho_{m}\right)+\nabla \cdot\left(\rho_{m} \bar{v}_{m}\right)=0,
$$

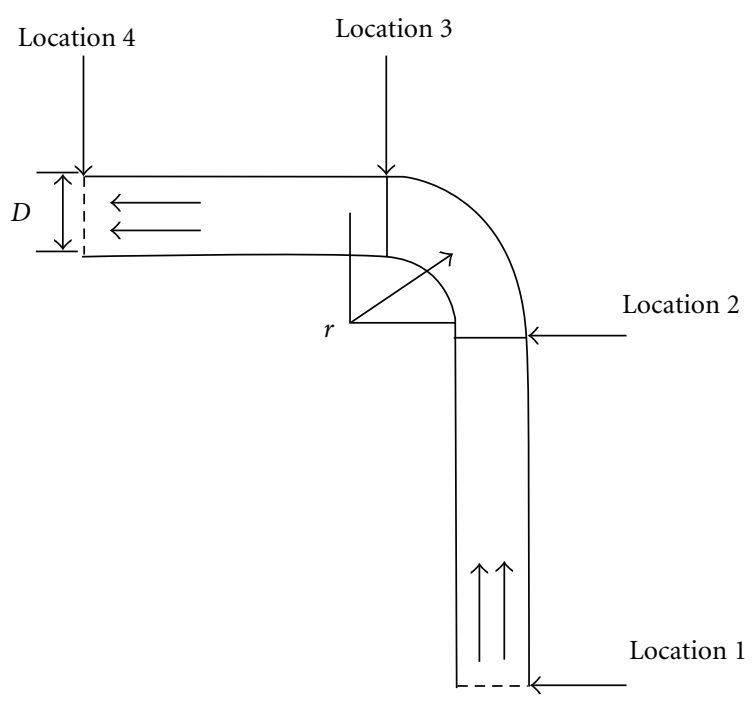

FIGURE 2: Locations of CFD and experimental data.

where the mass-averaged velocity $\left(\bar{v}_{m}\right)$ is

$$
\bar{v}_{m}=\frac{\sum_{k=1}^{n} \alpha_{k} \rho_{k} \bar{v}_{k}}{\rho_{m}},
$$

and the mixture density $\left(\rho_{m}\right)$ is given by

$$
\rho_{m}=\sum_{k=1}^{n} \alpha_{k} \rho_{k}
$$

where $\alpha_{k}$ is volume fraction of phase $k$.

By adding the individual momentum equations for all phases, the mixture's final momentum equation can be obtained, and it is given by

$$
\begin{aligned}
\frac{\partial}{\partial t}\left(\rho_{m} \bar{v}_{m}\right) & +\nabla \cdot\left(\rho_{m} \bar{v}_{m} \bar{v}_{m}\right) \\
= & -\nabla p+\nabla \cdot\left[\mu_{m}\left(\nabla \bar{v}_{m}+\nabla \bar{v}_{m}^{T}\right)\right] \\
& +\rho_{m} \bar{g}+\bar{F}+\nabla \cdot\left(\sum_{k=1}^{n} \alpha_{k} \rho_{k} \bar{v}_{d r, k} \bar{v}_{d r, k}\right),
\end{aligned}
$$

where $\bar{F}$ is the body force, $n$ is the number of phases, and $\mu_{m}$ is the viscosity of the mixture:

$$
\mu_{m}=\sum_{k=1}^{n} \alpha_{k} \mu_{k}
$$

where $\bar{v}_{d r, k}$ is the drift velocity for the secondary phase $k$ :

$$
\bar{v}_{d r, k}=\bar{v}_{k}-\bar{v}_{m}
$$

The relative velocity, or the slip velocity, is defined as the velocity of a secondary phase $(p)$, relative to the velocity of the primary phase $(q)$, and can be given by

$$
\bar{v}_{p q}=\bar{v}_{p}-\bar{v}_{q} .
$$


The mass fraction for any phase $(k)$ is defined as

$$
c_{k}=\frac{\alpha_{k} \rho_{k}}{\rho_{m}} .
$$

The drift velocity and the relative velocity $\left(\bar{v}_{p q}\right)$ are connected by the following expression:

$$
\bar{v}_{d r, p}=\bar{v}_{p q}-\sum_{k=1}^{n} c_{k} \bar{v}_{q k} .
$$

The algebraic slip formulation is used in the FLUENT's mixture model. Prescribing an algebraic relation for the relative velocity, a local equilibrium between phases should be reached over short spatial length scale, according to the basic assumption of the algebraic slip mixture model. The relative velocity then is given by

$$
\bar{v}_{p q}=\frac{\tau_{p}}{f_{\mathrm{drag}}} \frac{\left(\rho_{p}-\rho_{m}\right)}{\rho_{p}} \bar{a},
$$

where the particle relaxation time $\left(\tau_{p}\right)$ is given by

$$
\tau_{p}=\frac{\rho_{p} d_{p}^{2}}{18 \mu_{q}},
$$

$d$ is the diameter of the particles (or droplets or bubbles) of secondary phase, and $\bar{a}$ is the secondary-phase particle's acceleration. The default drag function $f_{\text {drag }}$ is taken from Schiller and Naumann [19]:

$$
f_{\text {drag }}= \begin{cases}1+0.15 \operatorname{Re}^{0.687} & \operatorname{Re} \leq 1000 \\ 0.0183 \operatorname{Re} & \operatorname{Re}>1000\end{cases}
$$

and the acceleration $\bar{a}$ is of the form

$$
\bar{a}=\bar{g}-\left(\bar{v}_{m} \cdot \nabla\right) \bar{v}_{m}-\frac{\partial \bar{v}_{m}}{\partial t} a .
$$

In the drift flux model, the acceleration of the particle is given by gravity and/or a centrifugal force, and, in order to take into account the presence of other particles, the particulate relaxation time is modified. In turbulent flows, the relative velocity should contain a diffusion term, due to the dispersion appearing in the momentum equation for the dispersed phase. FLUENT adds this dispersion to the relative velocity:

$$
\bar{v}_{p q}=\frac{\left(\rho_{p}-\rho_{m}\right) d_{p}^{2}}{18 \mu_{q} f_{\text {drag }}} \bar{a}-\frac{v_{m}}{\alpha_{p} \sigma_{D}} \nabla \alpha_{q},
$$

where $\left(v_{m}\right)$ is the mixture turbulent viscosity, and $\left(\sigma_{D}\right)$ is the Prandtl dispersion coefficient.

\section{Modeling Assumptions}

A straight pipe section was extended at the inlet and outlet boundary to evaluate the pressure drop across each elbow. In order to ensure fully developed flow, appropriate Le/D ratio was used to calculate the length of the straight section.
The standard $k-\epsilon$ model, with wall functions, was used in this study, since it is the simplest of the "complete models" available in FLUENT. The model constants used for this analysis were $C_{1 \epsilon}=1.44, C_{2 \epsilon}=1.92, C_{\mu}=0.09$, $\sigma_{k}=1.0$, and $\sigma_{\epsilon}=1.3$. For the mixture parameters, slip velocity was taken into consideration, as the phases had a significant difference in velocities, while the noslip boundary conditions were assumed for the wall of tubing. Due to the complex behavior of the two-phase flow, solution strategies were followed to improve the accuracy and convergence of the solution. The mixture calculation was initialized with a low under relaxation factor of 0.2 for the slip velocity; calculations were performed by combinations of the SIMPLE pressure-velocity coupling. The first-order upwind discretization scheme was used for the momentum, volume fraction, turbulent kinetic energy, and turbulent dissipation rate. The convergence criterion was based on the residual values of the calculated variables, to ensure satisfactory accuracy, stability, and convergence. The governing equations were solved sequentially, separate from one another, requiring less memory in comparison with the coupled algorithm. SIMPLE uses the pressure-based segregated algorithm, which makes use of the relationship between the velocity and pressure corrections, to enforce mass conservation, and to obtain the pressure field.

\section{CFD Analysis}

CFD analysis was performed on four different elbows, at nine different water and air velocities. Thus, a total of twentyseven different combinations of water and air velocities were used in the study, for each elbow. Each of these conditions was analyzed in CFD in order to accurately predict the effect of varying the pipe diameter and $r / D$ ratios. Due to the limitation of the test loop system, experimental investigations could not be performed for all of these conditions. Table 3 lists all the flow conditions that were used in the pressure loss CFD analysis. Numbers 1, 2, 3, and 4 in Table 3 represent elbows 1, 2, 3, and 4, respectively.

Cross-sectional absolute pressure and radial velocity contours are presented in Figure 3. The left side of Figure 3 shows the radial velocity contours in four locations of elbow 1 at a water velocity of $0.1 \mathrm{~m} / \mathrm{s}$ and air velocities of 15.24 , 30.48 , and $45.72 \mathrm{~m} / \mathrm{s}$, respectively. Location 1 represents the inlet of the upstream pipe, while location 2 indicates the outlet of the upstream pipe and inlet of the elbow. Similarly, location 3 indicates the outlet of elbow and inlet of the downstream pipe, and location 4 indicates the outlet of the downstream pipe. The top part of each individual contour represents the inside wall, while the bottom part of the contour represents the outer wall of the elbow. The contour maps were collected to locate, and study, any patterns that were formed. As depicted in Figure 3, the secondary flow pattern can be observed at the exit of the elbow section. The right side of Figure 3 shows the absolute pressure profiles in the four locations of elbow 1 at a water velocity of $0.1 \mathrm{~m} / \mathrm{s}$, and air velocities of $15.24,30.48$, and $45.72 \mathrm{~m} / \mathrm{s}$, respectively. The pressure distribution is dispersed without forming any regular pattern. 
TABLE 3: Flow conditions used in CFD analysis.

\begin{tabular}{|c|c|c|c|c|c|c|c|c|c|}
\hline \multirow{2}{*}{ Air velocity, $(\mathrm{m} / \mathrm{sec})$} & \multicolumn{9}{|c|}{ Water velocity, $(\mathrm{m} / \mathrm{sec})$} \\
\hline & 0.1 & 0.5 & 1.0 & 1.3 & 2.0 & 2.5 & 5.0 & 7.5 & 10.0 \\
\hline 15.24 & $1,2,3,4$ & 1,2 & $1,2,3,4$ & 1,2 & $1,2,3,4$ & 1,2 & $1,2,3,4$ & $1,2,3,4$ & $1,2,3,4$ \\
\hline 30.48 & $1,2,3,4$ & 1,2 & $1,2,3,4$ & 1,2 & $1,2,3,4$ & 1,2 & $1,2,3,4$ & $1,2,3,4$ & $1,2,3,4$ \\
\hline 45.72 & $1,2,3,4$ & 1,2 & $1,2,3,4$ & 1,2 & $1,2,3,4$ & 1,2 & $1,2,3,4$ & $1,2,3,4$ & $1,2,3,4$ \\
\hline
\end{tabular}

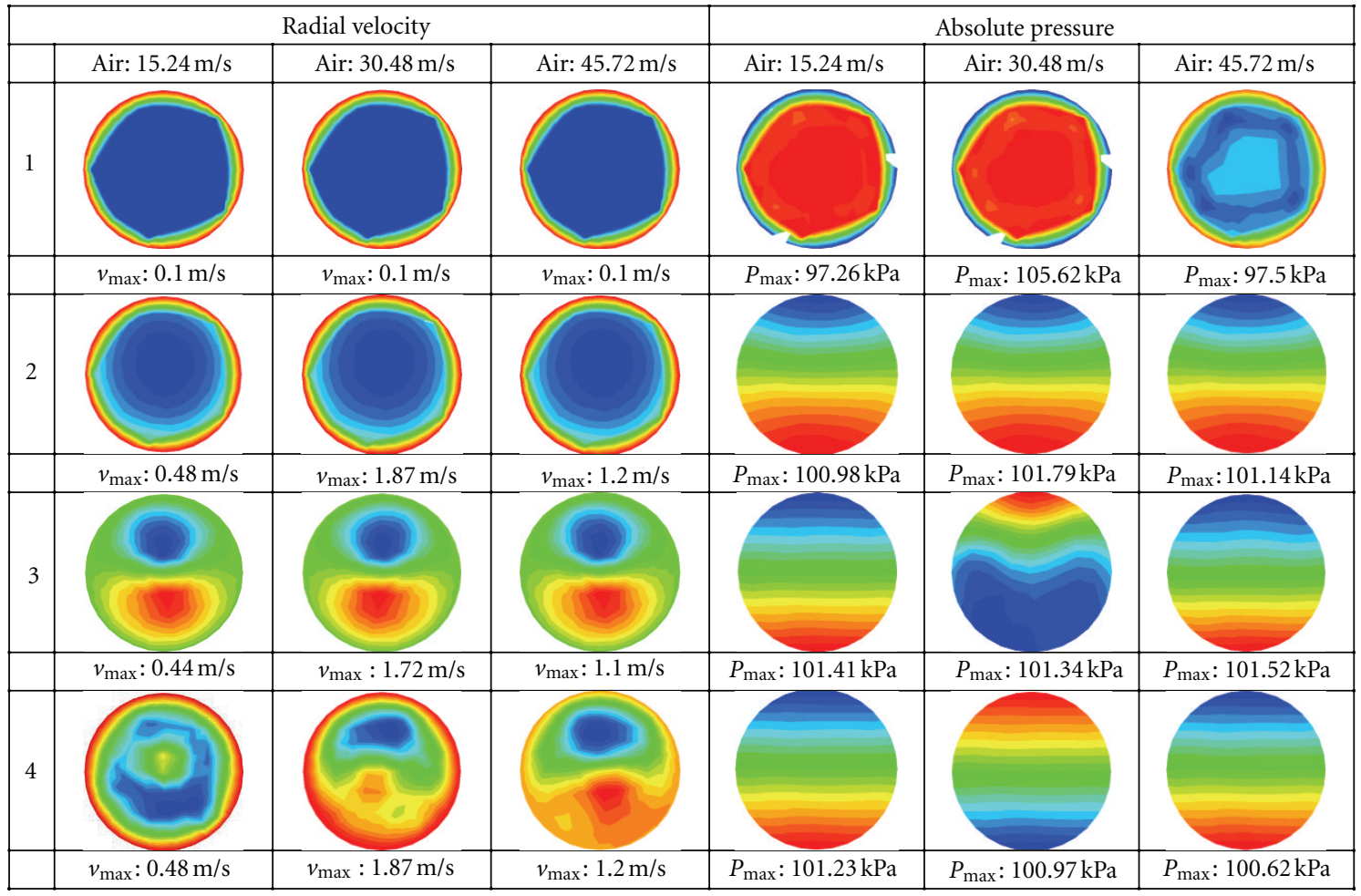

FIGURE 3: CFD-predicted velocity and pressures in elbow 1 at water $0.1 \mathrm{~m} / \mathrm{s}$.

\section{Pressure Drop Calculations with Empirical Models}

9.1. Chisholm Model. Chisholm proposed a correlation that involves the dimensionless parameters obtained by correlating two-phase flow experimental data [10]. It also requires an equivalent pipe length $L e$ that depends on the elbow radius to the pipe diameter ratio $(r / D)$, as well as the angle of the elbow bend [23]. The increment of $L e$ as a function of $r / D$ is mainly due to friction, centrifugal force, and the secondary flow that is present in the elbows. Thus, the single-phase pressure drop in the elbow is

$$
\Delta P_{1 \mathrm{ph}}=f \frac{G^{2}}{2 \rho}\left(\frac{L e}{D}\right)
$$

Chisholm suggested evaluating the pressure drop coefficient $k_{l}$, by assuming that the whole two-phase flow mixture flows as liquid only through the pipe fitting, as expressed by

$$
k_{l}=f_{l}\left(\frac{L e}{D}\right)
$$

Considering that only liquid phase fills up the pipe, the twophase flow mixture pressure drop is evaluated as

$$
\Delta P_{1 \mathrm{ph}, \mathrm{l}}=\frac{k_{l} G_{T}^{2}}{2 \rho_{l}} .
$$

Therefore, pressure drop in a two-phase mixture flowing through a $90^{\circ}$ elbow is given by an equation that already includes the mass quality, and a correlation factor, for twophase properties:

$$
\Delta P_{2 \mathrm{ph}}=\Delta P_{1 \mathrm{ph}, 1}\left[1+\left\{E(x(1-x))+x^{2}\right\}\right]
$$

where $E$ is $90^{\circ}$ elbow coefficient, which includes the relative radius of the elbow

$$
E=1+\frac{2.2}{k_{l}(2+(r / D))} .
$$

9.2. Azzi-Friedel Model. According to this model, the pressure drop is based on the two-phase flow multiplier, defined as the ratio of the bend pressure loss in two-phase flow, and 


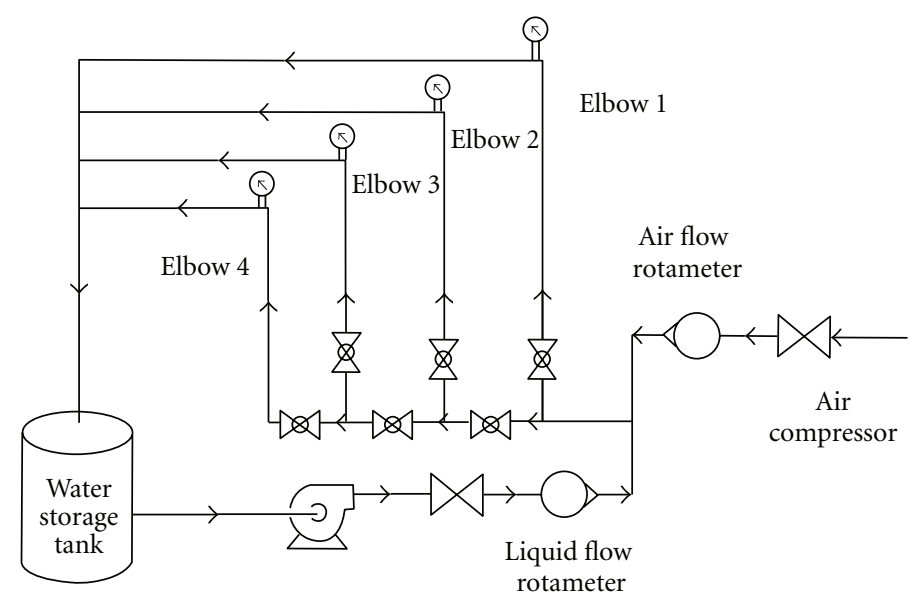

FIgURE 4: Schematic of the experimental test system.

that in the single-phase liquid flow with the same total mass flow rate as in [9]:

$$
\Phi^{2}=\frac{\Delta P_{2 \mathrm{ph}}}{\Delta P_{1 \mathrm{ph}, \mathrm{l}}}
$$

where $\Delta P_{1 \mathrm{ph}, \mathrm{l}}$ is the pressure drop of single-phase liquid fluid, across the same bend, defined as

$$
\Delta P_{1 \mathrm{ph}, \mathrm{l}}=k_{i} \frac{G_{l}^{2}}{2 \rho_{l}},
$$

where

$$
k_{i}=f_{i}\left(\frac{L e}{D}\right)
$$

where $L e / D$ is the dimensionless, single-phase equivalent length, and $f_{i}$ is the single-phase flow pipe friction factor. According to Churchill [24], this factor can be calculated by using the following equation:

$$
f_{i}=8\left(\left(\frac{8}{\operatorname{Re}}\right)^{12}+\left(A_{i}+B_{i}\right)^{-1.5}\right)^{1 / 12}
$$

where

$$
\begin{gathered}
A_{i}=\left(2.457 \ln \left(\left(\frac{7}{\operatorname{Re}_{i}}\right)^{0.9}+0.27 \frac{\varepsilon}{D}\right)^{-1}\right)^{16}, \\
B_{i}=\left(\frac{37530}{\operatorname{Re}_{i}}\right)^{16}, \\
\operatorname{Re}_{i}=\frac{\rho_{i} V_{i} D}{\mu_{i}} .
\end{gathered}
$$

Subscript $i$ in the above equations can be used for either liquid or gas.
The two-phase flow multiplier, defined by Azzi and Friedel, is given by

$$
\begin{gathered}
\Phi^{2}=C+7.42 F r_{l}^{0.125} \frac{r}{D}^{0.502} x^{0.7}(1-x)^{0.1} \\
\times\left(\frac{\left(\rho_{l}-\rho_{g}\right)}{\rho_{l}}\right){ }^{0.14}\left(\frac{\left(\mu_{l}-\mu_{g}\right)}{\mu_{l}}\right)^{0.12}, \\
C=(1-x)+\left(\frac{\rho_{l} k_{g}}{\rho_{g} k_{l}}\right) x^{2} .
\end{gathered}
$$

Froude number $\left(F r_{l}\right)$ is

$$
F r_{l}=\frac{\left(1-x^{2}\right) G_{T}^{2}}{\rho_{l}^{2} r g} .
$$

\section{Experimental Investigation}

To validate the CFD simulation and empirical model results, a multiphase air-water test system was designed and developed. Two different $12.7 \mathrm{~mm}$ and two different $6.35 \mathrm{~mm}$ test sections were used to conduct the pressure drop experiments. The major difference between each test section is the flow development length upstream of the test section, pipe diameter, and elbow radius to pipe diameter ratio. Figure 4 shows the schematic of the test loop, consisting of a 30-gallon water tank, a 25 HP pump, a 10 CFM air compressor, liquid and gas flow meters, four vertical to horizontal $90^{\circ}$ elbows with two different pipe diameters and $r / D$ ratios, inlet and outlet pressure gauges, and four differential pressure gauges. Air from the compressor enters the test loop, through a gate valve and air flow meter, that is used to control the air flow rates. Water from the tank is pumped into the test section, through a gate valve and liquid flow meter, that is used to control the water flow rates. Water is injected into the air stream through a $T$ elbow, and the mixture then flows through a straight section of $12.7 \mathrm{~mm}$ pipe section to the elbows. Ball valves are used to allow the mixture to enter a specific elbow and return the water to tank. The $12.7 \mathrm{~mm}$ and 


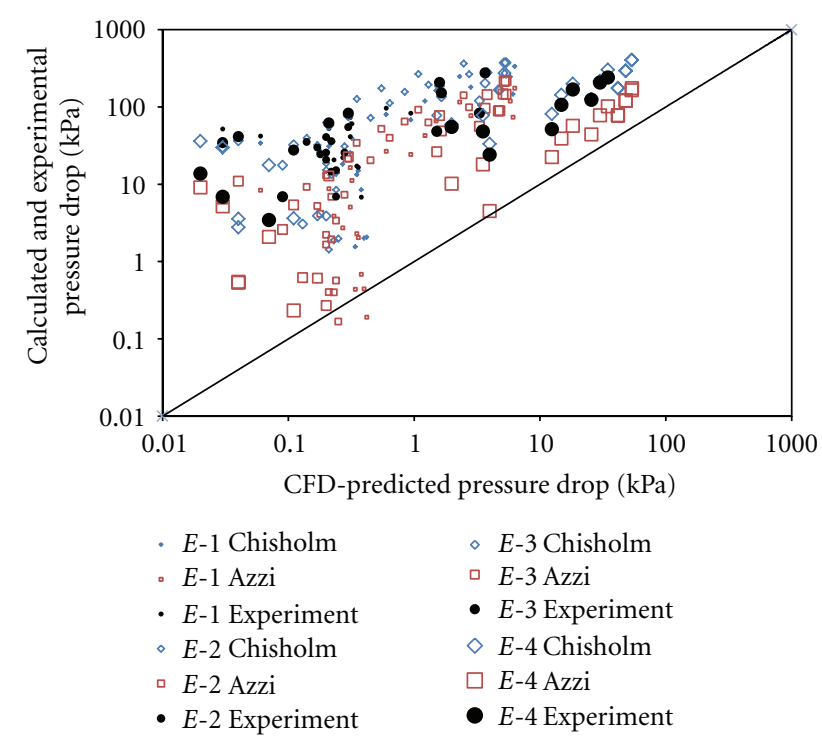

Figure 5: Comparison of CFD versus calculated and experimental pressure drop.

$6.35 \mathrm{~mm}$ transparent sections of plexiglass pipe are used for multiphase flow pattern visualization. The pressure drop was measured across each elbow, using the differential pressure gauges hooked up across the 90 degree elbows. After the test sections, the mixture flows downstream, into the water tank, where the mixture is separated. The air is released back into the environment, while the water is recycled.

\section{Comparison of CFD Results with Experimental and Empirical Results}

Figure 5 shows the plot of CFD-predicted pressure drop versus two empirical and experimental pressure drop data for all elbows. The linear line shown in Figure 5 depicts a perfect line. The data above the line is overpredicted results, while the data below the line is underpredicted results. The data lying near the line shows perfect agreement. In all conditions, CFD underpredicted the pressure drop values. Azzi and Friedel, Chisholm, and the experiment overpredicted the pressure drop. However, for elbows 1 and 2, Azzi-Friedel's model underpredicted the pressure drop. On the same token, Chisholm model significantly over predicted the pressure drop for elbows 1, 2 and 3, which can be seen in Figure 5. For both empirical models, the prediction for elbows 3 and 4 was closer to the perfect agreement line.

\section{Summary and Conclusion}

CFD analysis of two-phase flow in a 6.35 , and $12.7 \mathrm{~mm}$ pipe diameter with $r / D$ ratio of 1.5 and 3 was performed using commercially available CFD code FLUENT. Analysis was performed for three different air velocities between $15.24,30.48$, and $45.72 \mathrm{~m} / \mathrm{s}$ and six different water velocities, ranging from 0.1 to $10.0 \mathrm{~m} / \mathrm{s}$, in each of the four elbows. Pressure drop profiles and their respective cross-sectional pressure contour maps were presented for characteristic flow behaviors in multiphase flows.

\section{Nomenclature}

English Letters

1-x: Wetness fraction/liquid quality

$A$ : Coefficient 1 of pipe friction factor

a: Acceleration

B: $\quad$ Coefficient 2 of pipe friction factor

C: $\quad$ Coefficient of two-phase multiplier

$D$ : Inner pipe diameter

E: $\quad$ Chisholm coefficient

$f$ : $\quad$ Friction factor for pipe

F: $\quad$ Force

$F r: \quad$ Froude number

G: $\quad$ Mass flux

g: Gravitational acceleration

$K: \quad$ Friction factor for bend

$L e / D$ : Equivalent pipe length to pipe diameter ratio

$r$ : $\quad$ Elbow curvature radius

$r / D$ : Elbow curvature radius to pipe diameter ratio

Re: Reynolds number

$v$ : Velocity

$x$ : Dryness fraction/gas quality

$\Delta P: \quad$ Two-phase pressure drop.

\section{Greek Letters}

$\mu$ : Dynamic viscosity

$\alpha$ : Volume fraction

$\varepsilon / D$ : Equivalent roughness to pipe diameter ratio

$\rho:$ Density

$\sigma:$ Prandtl coefficient

$\tau$ : Relaxation time

$\Phi^{2}$ : Two-phase multiplier.

\section{Subscripts}

1ph: Single-phase flow

2ph: Two-phase flow

$d$ : Dispersion

$d r:$ Drift

drag: Drag force

$e$ : Equivalent

g: Gas phase

$i$ : Subscript for either liquid or gas phase

$k$ : Any phase

$l: \quad$ Liquid phase

$m$ : Mixture

$p: \quad$ Secondary phase

pq: Relative phase

q: $\quad$ Primary phase

T: Total

$t$ : Time. 


\section{References}

[1] S. F. Sánchez, R. J. C. Luna, M. I. Carvajal, and E. Tolentino:, "Pressure drop models evaluation for two-phase flow in 90 degree horizontal elbows," Ingenieria Mecanica Techilogia Y Desarrollo, vol. 3, no. 4, pp. 115-122, 2010.

[2] G. B. Wallis, One Dimensional Two-Phase Flow, McGraw-Hill, 1969.

[3] S. Benbella, M. Al-Shannag, and Z. A. Al-Anber, "Gasliquid pressure drop in vertical internally wavy $90^{\circ}$ bend," Experimental Thermal and Fluid Science, vol. 33, no. 2, pp. 340-347, 2009.

[4] J. S. Cole, G. F. Donnelly, and P. L. Spedding, "Friction factors in two phase horizontal pipe flow," International Communications in Heat and Mass Transfer, vol. 31, no. 7, pp. 909-917, 2004.

[5] S. Wongwises and W. Kongkiatwanitch, "Interfacial friction factor in vertical upward gas-liquid annular two-phase flow," International Communications in Heat and Mass Transfer, vol. 28, no. 3, pp. 323-336, 2001.

[6] P. L. Spedding, E. Benard, and G. F. Donnelly, "Prediction of pressure drop in multiphase horizontal pipe flow," International Communications in Heat and Mass Transfer, vol. 33, no. 9, pp. 1053-1062, 2006.

[7] J. Hernández Ruíz, Estudio del comportamiento de flujo de fluidos en tuberías curvas para plicaciones en metrología [Tesis de Maestría], IPN-ESIME, 1998.

[8] A. M. Chan, K. J. Maynard, J. Ramundi, and E. Wiklund, "Qualifying elbow meters for high pressure flow measurements in an operating nuclear power plant," in Proceedings of the 14th International Conference on Nuclear Engineering (ICONE '06), Miami, Fla, USA, July 2006.

[9] A. Azzi and L. Friedel, "Two-phase upward flow $90^{\circ}$ bend pressure loss model," Forschung im Ingenieurwesen, vol. 69, no. 2, pp. 120-130, 2005.

[10] D. Chisholm, Two-Phase Flow in Pipelines and Heat Exchangers, Godwin, 1983.

[11] J. M. Chenoweth and M. W. Martin, "Turbulent two-phase flow," Petroleum Refiner, vol. 34, no. 10, pp. 151-155, 1955.

[12] R. W. Lockhart and R. C. Martinelli, "Proposed correlation of data for isothermal two-phase two-component flow in pipes," Chemical Engineering Progress, vol. 45, no. 1, pp. 39-48, 1949.

[13] P. E. Fitzsimmons, "Two phase pressure drop in pipe components,” Tech. Rep. HW-80970 Rev 1, General Electric Research, 1964.

[14] K. Sekoda, Y. Sato, and S. Kariya, "Horizontal two-phase airwater flow characteristics in the disturbed region due to a 90degree bend," Japan Society Mechanical Engineering, vol. 35, no. 289, pp. 2227-2333, 1969.

[15] A. Asghar, R. Masoud, S. Jafar, and A. A. Ammar, "CFD and artificial neural network modeling of two-phase flow pressure drop," International Communications in Heat and Mass Transfer, vol. 36, no. 8, pp. 850-856, 2009.

[16] T. L. Deobold, "An experimental investigation of two-phase pressure losses in pipe elbows," Tech. Rep. HW-SA, 2564, MSc. University of Idaho, Chemical Engineering, 1962.

[17] G. E. Alves, "Co-current liquid-gas flow in a pipe-line contactor," Chemical Engineering Progress, vol. 50, no. 9, pp. 449-456, 1954.

[18] M. A. Peshkin, "About the hydraulic resistance of pipe bends to the flow of gas-liquid mixtures," Teploenergetika, vol. 8, no. 6, pp. 79-80, 1961.

[19] S. S. Kutateladze, Problems of Heat Transfer and Hydraulics of Two-Phase Media, Pergamon Press, Oxford, UK.
[20] S. F. Moujaes and S. Aekula, "CFD predictions and experimental comparisons of pressure drop effects of turning vanes in $90^{\circ}$ duct elbows," Journal of Energy Engineering, vol. 135, no. 4, pp. 119-126, 2009.

[21] Q. H. Mazumder, S. A. Shirazi, and B. S. McLaury, "Prediction of solid particle erosive wear of elbows in multiphase annular flow-model development and experimental validation," Journal of Energy Resources Technology, vol. 130, no. 2, Article ID 023001, 10 pages, 2008.

[22] I. Fluent, Fluent 6. 3 User Guide, Fluent Inc., Lebanon, NH, USA, 2002.

[23] N. P. Cheremisinoff, Ed., Encyclopedia of Fluid Mechanics: Gas Liquid Flows, vol. 3, Gulf Publishing Company, 1986.

[24] S. W. Churchill, "Friction equation spans all fluid flow regimes," Chemical Engineering, vol. 84, no. 24, pp. 91-92, 1977. 

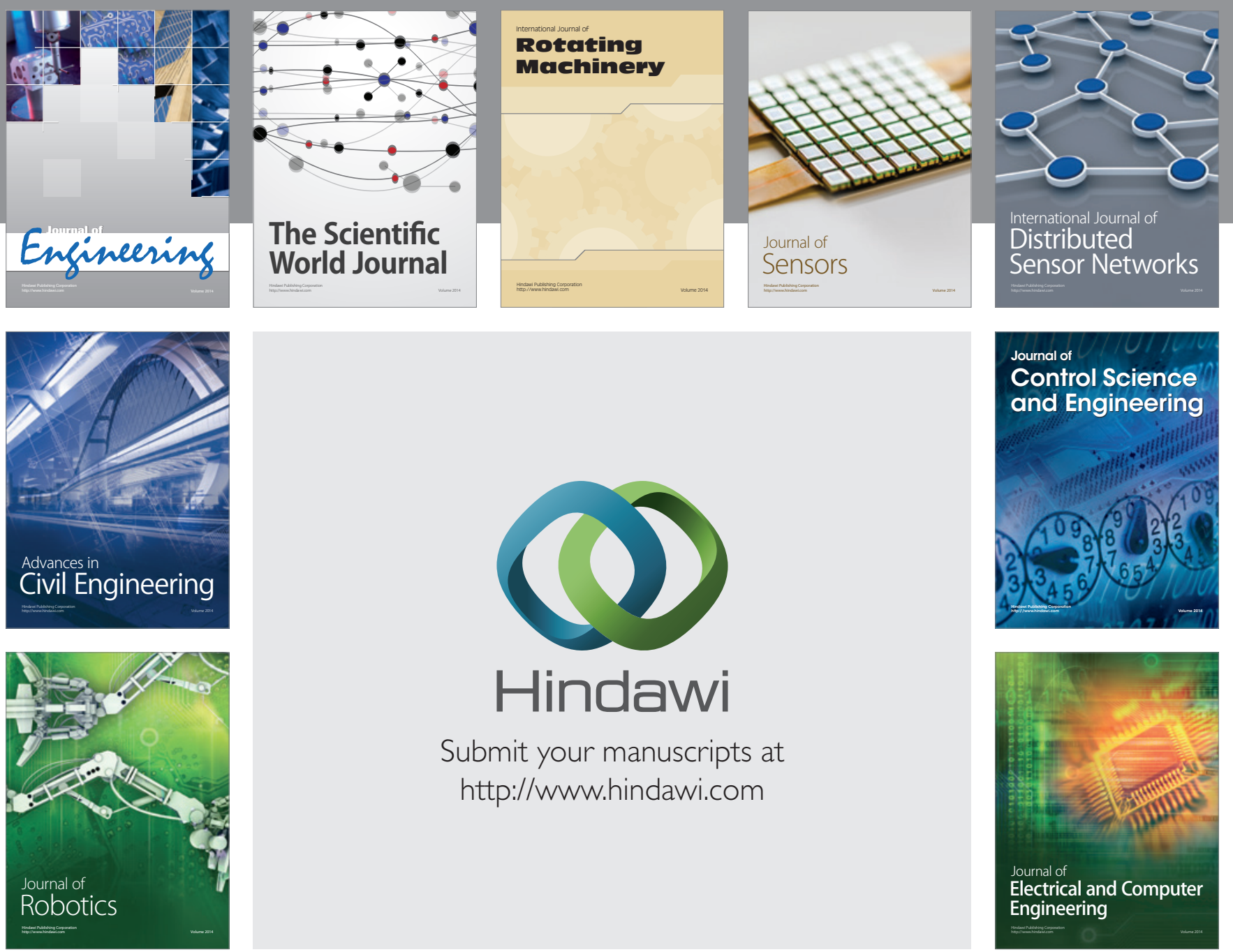

Submit your manuscripts at

http://www.hindawi.com
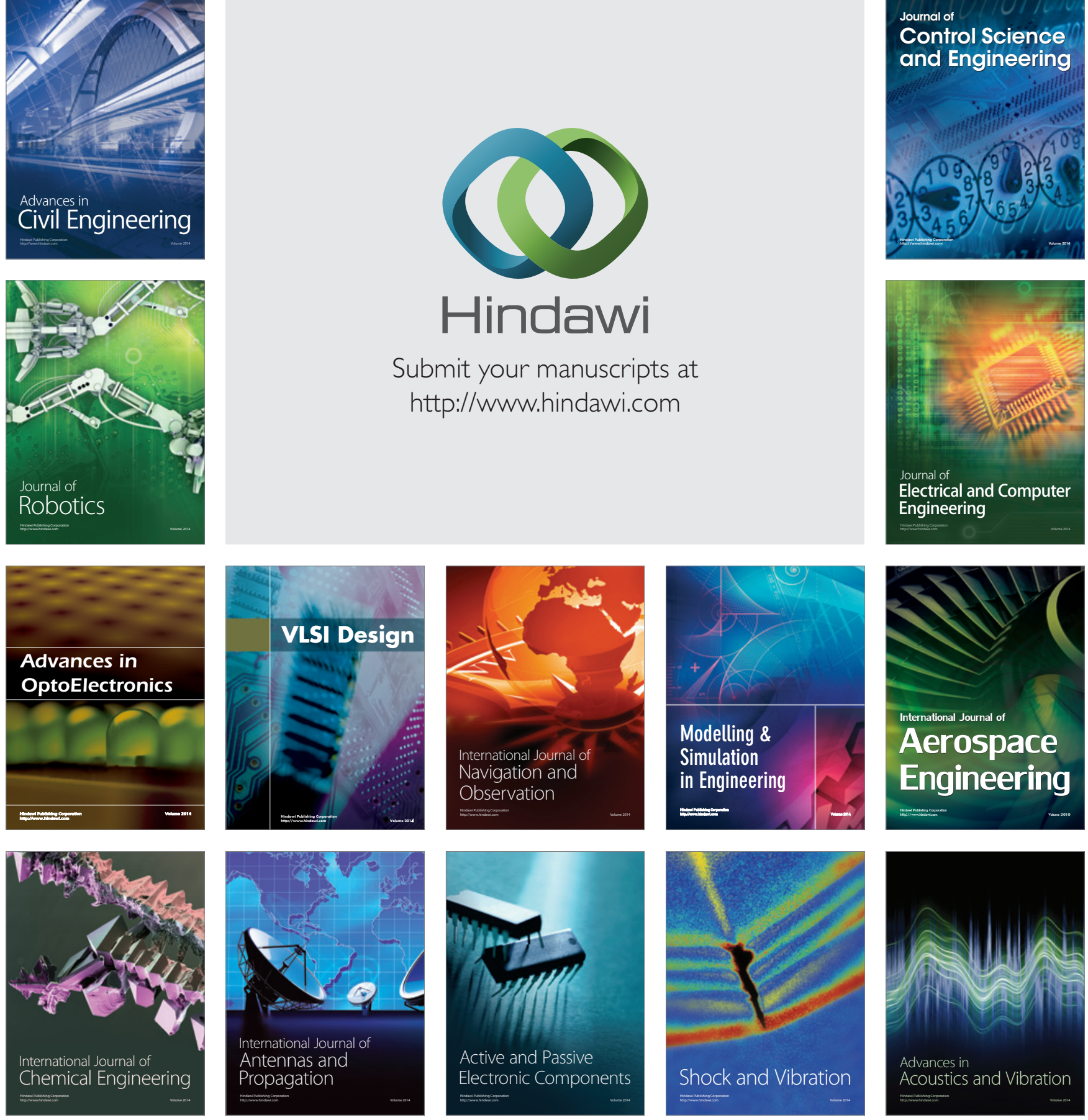